This item was submitted to Loughborough's Research Repository by the author.

Items in Figshare are protected by copyright, with all rights reserved, unless otherwise indicated.

\title{
On the assessment by grazing-incidence small-angle X-ray scattering of replica quality in polymer gratings fabricated by nanoimprint lithography
}

\section{PLEASE CITE THE PUBLISHED VERSION}

http://dx.doi.org/10.1107/S160057671400168X

\section{PUBLISHER}

(c) International Union of Crystallography

\section{VERSION}

AM (Accepted Manuscript)

\section{PUBLISHER STATEMENT}

This work is made available according to the conditions of the Creative Commons Attribution-NonCommercialNoDerivatives 4.0 International (CC BY-NC-ND 4.0) licence. Full details of this licence are available at: https://creativecommons.org/licenses/by-nc-nd/4.0/

\section{LICENCE}

CC BY-NC-ND 4.0

\section{REPOSITORY RECORD}

Soccio, M., N. Alayo, Ignacio Martin-Fabiani, Daniel R. Rueda, Mari-Cruz Garcia-Gutierrez, Esther Rebollar, D.E. Martinez-Tong, F. Perez-Murano, and Tiberio A. Ezquerra. 2017. "On the Assessment by Grazingincidence Small-angle X-ray Scattering of Replica Quality in Polymer Gratings Fabricated by Nanoimprint Lithography”. figshare. https://hdl.handle.net/2134/26699. 
On the Assessment by Grazing Incidence Small Angle X-ray Scattering of Replica Quality in Polymer Gratings Fabricated by Nanoimprint Lithography

\author{
M. Soccio ${ }^{1}$, N. Alayo $^{2}$, I. Martín-Fabiani ${ }^{1}$, D.R. Rueda ${ }^{1}$, M.C. García-Gutiérrez ${ }^{1}$, E. \\ Rebollar $^{3}$, D. Martínez-Tong ${ }^{1}$, F. Pérez-Murano ${ }^{2}$, T.A. Ezquerra ${ }^{1}$ \\ ${ }^{1}$ Instituto de Estructura de la Materia, IEM-CSIC \\ Serrano 119, 121, Madrid 28006, Spain \\ 2 Instituto de Microelectrónica de Barcelona IMB-CNM-CSIC, Campus UAB 08193, \\ Bellaterra, Spain \\ ${ }^{3}$ Instituto de Química Física Rocasolano, IQFR-CSIC, Serrano 119, 28006 Madrid, \\ Spain
}

\begin{abstract}
Grazing Incidence Small Angle X-ray Scattering (GISAXS) can be used in an attempt to characterize replica quality of polymer gratings prepared by thermal Nanoimprint Lithography (NIL). Here we show that GISAXS experiments performed in a series of NIL polymer gratings with different line quality present characteristic features that can be associated with the level of defects per line. Both, stamps and NIL polymer gratings exhibit characteristic semicircle-like GISAXS patterns. However NIL polymer gratings with defective lines exhibit GISAXS patterns with an excess of diffuse scattering as compared to those of the corresponding stamps. In a first approach we attribute this effect to a reduction of the effective length of the lines diffracting coherently as the amount of defects per line increases.
\end{abstract}




\section{Introduction}

Nanoimprint lithography (NIL) is a straightforward, high-throughput and highresolution patterning technique in which a stamp, with a certain surface pattern, is replicated onto a material by mechanical contact(Schift, 2008). When the material to be patterned is a thermoplastic polymer, NIL can be performed by varying the thermomechanical properties of the material by heating and cooling. Typically, a hard mold with a micro/nanofabricated pattern on its surface is pressed into a polymer. The polymer, prepared as a thick slice or spin-coated onto a rigid or flexible substrate, has to be heated either above its glass transition temperature ( $\left.\mathrm{T}_{\mathrm{g}}\right)$ (Schift, 2008; Cecchini et al., $2008)$, in the case of an amorphous polymer, or above its melting temperature $\left(T_{m}\right)$ for a semicrystalline one (Hu et al., 2009; Hu \& Jonas, 2010). The mold can be removed after the polymer film has been cooled down below $\mathrm{T}_{\mathrm{g}}$. This NIL procedure is frequently referred to as hot embossing lithography or thermal NIL (Schift, 2008; Hu \& Jonas, 2010). NIL can achieve resolutions beyond the limitations set by light diffraction that are encountered in other traditional techniques. In particular, NIL enables controlled surface modification of polymer materials for which standard lithographic schemes are not applicable due to incompatibility with developers, organic solvents, etc. The patterning of flexible polymer substrates in the micro and nanometer scale enable new applications in the area of photonics (Lochbihler, 2009), photovoltaics (Mayer et al., 2007; Chen et al., 2012; Meier et al., 2012a) and biology (Lee, 2006; Mills et al., 2005) among others. Structural characterization of NIL patterned polymers is commonly accomplished by microscopy techniques. The visualization in real space has obvious advantages since it provides access to relevant morphological information. However, quite frequently visualization is restricted to small and superficial areas of the sample. On the contrary, X-ray scattering techniques provide structural information in the reciprocal space. The diffraction pattern reveals information averaged over a large sample volume covered by the footprint of the incident beam on the material surface and enables time-resolved experiments. Consequently, diffraction can provide supplementary information to microscopy. In order to perform X-ray diffraction on surface patterned polymers it is very convenient to work under total X-ray reflection conditions(Müller-Buschbaum, 2006). The technique of Grazing Incidence Small-Angle X-ray Scattering (GISAXS) has already been applied to characterize structurally a great variety of polymer gratings (Rueda et al., 2012; Meier et al., 2012b; Hlaing et al., 
2012). Although being NIL an established technique, there is still little understanding about the physics underlying the imprint process. In this respect, computer simulation is currently being used in an attempt to acquire basic knowledge about physical mechanisms involved in NIL (Dirckx \& Hardt, 2011; Chandross \& Grest, 2012). In general, the filling of the mold by the polymer typically involves squeezed-flow and complex shear forces. In addition, demolding by detachment of the stamp from the solidified polymer involves complex friction forces, displacement of air and capillary bridges that typically may produce damages on the nanoimprinted pattern (Schift, 2008). The main aim of this work is to discuss the potential of GISAXS for the assessment of replica quality in some model polymer gratings fabricated by NIL with special emphasis to the surface damage induced by demolding.

\section{Experimental part}

\subsection{Polymer Materials}

Poly(bisphenol A carbonate) (PBAC) (Lexan ML3021A,SABIC I-P(Innovative Plastics), $M_{w}=44.4 \times 10^{3} \mathrm{~g} / \mathrm{mol}, M_{n}=23.5 \times 10^{3} \mathrm{~g} / \mathrm{mol}$ (as revealed by size-exclusion chromatography) has been used as a model amorphous polymer. PBAC exhibits a glass transition temperature $\mathrm{T}_{\mathrm{g}}=145^{\circ} \mathrm{C}$, as determined by calorimetry. PBAC is a commodity polymer broadly used for everyday life applications. Thin films were prepared by spin coating on silicon wafers (100) (Wafer World Inc.) polished on one surface. The wafers were previously cleaned with acetone and isopropanol respectively, and dried by nitrogen flow. PBAC was dissolved in chloroform (Sigma-Aldrich, reagent grade $\geq 98 \%$ ), with a concentration of $20 \mathrm{~g} / \mathrm{L}$. A fixed amount of $0.1 \mathrm{~mL}$ of polymer solution was deposited by a syringe on a square $\left(2 \times 2 \mathrm{~cm}^{2}\right)$ silicon substrate placed in the centre of the metallic horizontal plate of the spinner. A rotation speed of $3000 \mathrm{rpm}$ was reached after a $0.1 \mathrm{~s}$ acceleration period and kept for 1 minute. Polymer films about $150 \pm 20 \mathrm{~nm}$ thick, as measured by spectrophotometry (Nanospec 6100), are typically obtained under these conditions.

\subsection{Nanofabrication of gratings on silicon stamps}

Hard gratings on silicon were prepared with two different pitches, L=200 nm and $\mathrm{L}=300 \mathrm{~nm}$, with line widths $\mathrm{D}$ from $20 \%$ to $50 \%$ of the pitch. The arrays of lines and trenches were fabricated on mesa-type silicon stamps to facilitate the imprinting process. The mesa, typically with an area of $0.2 \times 0.2 \mathrm{~cm}^{2}$ and $400 \mathrm{~nm}$ height, was 
prepared by means of optical lithography and wet silicon etching (Fig.1a). On top of the mesa, arrays of lines 50-150 $\mathrm{nm}$ wide were defined by electron beam lithography (Raith 150 Two, Raith) on a $100 \mathrm{~nm}$ resist layer (Poly(methyl methacrylate) $950 \mathrm{~kg} / \mathrm{mol}$ ). The e-beam worked by exposing in writing fields of $200 \times 200 \mu \mathrm{m}^{2}$ (Fig. 1b). After the development of the resist in methyl isobutyl ketone the patterns were defined by deposition of $20 \mathrm{~nm}$ of aluminum using e-beam evaporation and lift-off in acetone at $40^{\circ} \mathrm{C}$. The resulting metal patterns were used as a mask for reactive ion etching (AMS $110 \mathrm{DE}$ ), using a mixture of $\mathrm{SF}_{6}$ and $\mathrm{C}_{4} \mathrm{~F}_{8}$ as reaction gases to achieve vertical profiles which allowed to obtain trenches of about $130 \pm 30 \mathrm{~nm}$ in between the lines (Fig.1c and 1d). Before the imprinting process, stamps were silanized with trichloro $(1 \mathrm{H}, 1 \mathrm{H}, 2 \mathrm{H}$, $2 \mathrm{H}$-perfluorooctyl)-Silanelane in order to avoid sticking of the polymer. Characterization of the stamps was performed by SEM (Leo 1530, Zeiss) and AFM (Nanoscope V and Dimension 3100, Bruker) in tapping mode. Upon dealing with relatively high aspect ratio nanostructures, the convolution of AFM tip shape with that of the sample provokes that parallel vertical walls may look slightly asymmetric.

\section{Preparation of Polymer gratings by NIL}

The silicon gratings were used as stamps to imprint spin-coated thin films of PBAC using a thermal nanoimprint system (Obducat). First, a temperature of $180^{\circ} \mathrm{C}$ was reached in about 3 minutes and in absence of pressure. Second, a nominal pressure of 40 atm was applied for 5 minutes. Third, the temperature was reduced to $80^{\circ} \mathrm{C}$, keeping the applied pressure. Fourth, pressure was removed and the system was allowed to gradually cool down to room temperature. Finally, the stamp was detached from the polymer nanostructure. Characterization of the polymer gratings was performed by an AFM (Nanoscope Multimode V, Bruker) in tapping mode.

\subsection{Grazing Incidence Small Angle X-ray Scattering.}

Polymer gratings were investigated by means of GISAXS using the BW4 beamline at HASYLAB (DORIS, DESY, Hamburg, Germany). The experimental set-up for GISAXS has been previously described(Roth et al., 2006; Timmann et al., 2009; Perlich et al., 2010; Hernandez et al., 2010) . An X-ray wavelength $\lambda=0.13808 \mathrm{~nm}$, with a beam size $(\mathrm{HxV})$ of $40 \times 20 \mu \mathrm{m}^{2}$ was used in our experiments. Scattered intensity 
was recorded by a Mar CCD detector of 2048x2048 pixels with a resolution of $79.1 \mu \mathrm{m}$ per pixel, and a sample-to-detector distance of $2.21 \mathrm{~m}$. Samples were positioned horizontally and were aligned with the lines parallel to the X-ray beam. Although a nominal incidence angle of $\alpha_{i}=0.40^{\circ}$ was selected, due to the small size of the samples $(\approx 1 \mathrm{~cm})$ alignment process rendered to experimental incidence angle values of $\alpha=0.37^{\circ} \pm 0.04^{\circ}$. Acquisition times were optimized in order to get maximum number of counts avoiding saturation of the detector. In the present case, typical acquisition times of $1 \mathrm{~s}$ for stamps and $5 \mathrm{~s}$ for NIL polymer gratings were used. In a GISAXS set-up the incoming beam and the sample surface normal define the vertical scattering plane (Müller-Buschbaum, 2009). Both the scattering plane and the sample plane intersect the detector along the meridian and the horizon lines respectively which are the reference to measure the horizontal $(\omega)$ and the $\operatorname{vertical}(\alpha)$ angles. The information can be interpreted on the basis of the three orthogonal scattering vectors $q_{z}=(2 \pi / \lambda)\left(\sin \alpha_{i}+\right.$ $\sin \alpha), q_{y}=(2 \pi / \lambda) \sin \omega \cos \alpha$ and $q_{x}=(2 \pi / \lambda)\left(\cos \omega \cos \alpha-\cos \alpha_{i}\right)$. While $q_{z}$ provides information about structural correlations perpendicular to the sample film, $q_{y}$ probes the correlations in horizontal known as in-plane correlations (Müller-Buschbaum, 2009). GISAXS images were analyzed by using the software Fit2D (Hammersley, 2004).

\section{Results}

Fig. 2 shows the morphology as revealed by AFM of some silicon stamps of similar pitch $\left(\mathrm{L}_{\text {stamp }}=300 \mathrm{~nm}\right)$ and different line widths $\left(\mathrm{D}_{\text {stamp }}\right)$ and heights $\left(\mathrm{H}_{\text {stamp }}\right)$ (upper panel) and the corresponding replicas in PBAC (bottom panel). The NIL polymer gratings are formed by parallel rectangular motives (lines) of different widths $\left(\mathrm{D}_{\text {nil }} \approx\right.$ $\mathrm{L}_{\text {stamp }}-\mathrm{D}_{\text {stamp}}$ ) and pitches similar to those of the stamps. Height profiles of both stamps and NIL polymer gratings are included. It is worth to remark the obvious decrease in surface quality of the NIL polymer gratings, as going from the left figure to the right one, most likely caused during demolding by imperfections of the different stamps. A qualitative evaluation of the extension of defects along a polymer line can be attempted by evaluating the ratio between the darker area, identified as defects, to the lighter area (non defective surface) by using a self made $\mathrm{MatLab}^{\odot}$ routine. An example of this procedure is illustrated in Fig. 3 for the polymer gratings shown in Fig.2.

Characteristic GISAXS patterns for the different silicon gratings investigated here are shown in Fig. 4 (top panel). As previously described, this type of silicon gratings exhibits as dominant feature the presence of spots on a semicircle which is related to the 
incidence angle used. Spots on the semicircle are accompanied by weaker scattering in the vertical direction. Spots are consecutive orders of the first one (nearest to $\omega=0^{\circ}$ ) whose spacing is related to the grating pitch. It has been previously reported that GISAXS patterns of hard gratings are due to the intersection of the Ewald sphere with the reciprocal space truncation rods characteristic of the grating (Metzger et al., 1997; Baumbach \& Lubbert, 1999; Mikulik et al., 2001; Yan \& Gibaud, 2007; Wernecke et al., 2012). Essentially, similar features are observed for the gratings nanofabricated by NIL on PBAC by using the silicon gratings as stamp (Fig. 4, bottom panel). However, it is worth mentioning that the NIL gratings exhibit, besides the characteristic scattering on semicircle, significant scattering around the angular region of the Yoneda peak (in our case $\alpha \approx 0.15-0.20^{\circ}$ ) associated with the diffuse scattering(Yoneda, 1963). It is worth mentioning that GISAXS patterns of non nanostructurated spin coated polymer films exhibit essentially no scattering features out of the scattering plane (Hernandez et al., 2010). This is the case for the PBAC spin coated thin films previous to the NIL process.

\section{Discussion.}

NIL is a highly dynamic process where the vertical sinking movement of a hard stamp is transformed into a three dimensional flow of the soft material with large lateral components. In thermal NIL, stamp cavities have to be filled by the polymer which has to flow over large distances. At the microscopic level, squeeze flow, displacement of air, and capillary bridges have to be considered (Schift, 2008; Dirckx \& Hardt, 2011; Rowland et al., 2005; Chandross \& Grest, 2012). In our case, in order to prepare polymer gratings, the mesa design with protruded motives (Fig.1d) is crucial for the success of the imprinting due to the concentration of the applied pressure in a small area. During NIL process, an amorphous polymer film must be heated up above its glass transition temperature and pressed. The polymer deformation occurs near the indenter sidewall, and the protrusions of the stamp generate the trenches in the polymer film. Each protruded line produces vertical and lateral flows of polymer material which interacts with the pressure generated by the adjacent lines. In Fig.5 we have plotted the ratio between the heights of the NIL polymer gratings to those of the corresponding stamps $\left(\mathrm{H}_{\mathrm{n}}=\mathrm{H}_{\text {nil }} / \mathrm{H}_{\text {stamp }}\right)$ as a function of the ratio of the indenter width to the pitch of the mold ( $\left.\mathrm{D}_{\text {stamp }} / \mathrm{L}_{\text {stamp }}\right)$. This magnitude is referred to as the filling factor of the stamp (Fernández Cuesta, 2009). It is worth to emphasize that this study was done for high quality NIL polymer gratings like the one shown in the inset of Fig.5 where $\mathrm{H}_{\text {nil }}$ could 
be properly determined. As one can see, the transfer of the stamp structure to the polymer film is more effective $\left(\mathrm{H}_{\mathrm{n}} \approx 1\right)$ for low values of $\mathrm{D}_{\text {stamp }} / \mathrm{L}_{\text {stamp. For a constant }}$

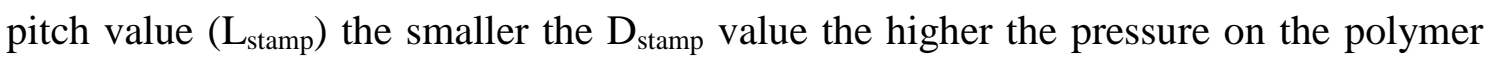
film beneath the indenter and the bigger is the cavity space where the polymer can flow. A similar effect was observed on NIL polymer gratings prepared with stamps of a lower pitch $L=200 \mathrm{~nm}$. Our results indicate that for values of $D_{\text {stamp }} / L_{\text {stamp }} \leq 0.3$, the lines of the stamp enter completely in the polymer substrate. For higher filling factors $\left(D_{\text {stamp }} / L_{\text {stamp }}>0.45\right)$, corresponding to larger line widths $\left(D_{\text {stamp }}\right)$ a lower pressure is exerted on the polymer film and the filling of cavities becomes less efficient. For the stamps shown in Fig.2 (upper panel) and the corresponding NIL gratings the filling factor is small $\left(\mathrm{D}_{\text {stamp }} / \mathrm{L}_{\text {stamp }} \leq 0.3\right)$ and therefore the filling of the stamp cavities by the polymer was very efficient. Accordingly, in a first approach, we attribute the defective surface (Figs. 2e and 2f) as caused during detaching by imperfections of the stamps. The quality of the stamp replication in the NIL polymer gratings can be assessed by comparison of their GISAXS patterns. Fig. 6 shows the total integrated intensity, along the vertical direction of the GISAXS patterns corresponding to the stamps and to the NIL polymer gratings shown in Fig.4. The integral intensity was preferred instead of a $\omega$-cut at a selected $\alpha$ value in order to capture all the characteristic features of the diffraction semicircle involving different $\alpha$-values. It can be seen that the similarity between the intensity profiles of the stamps (dotted) and those of the NIL gratings (continuous) is particularly good concerning periodicity. Thus, NIL polymer grating patterns reproduce rather well both the profile of scattered intensity and the periodicity of the gratings in spite of the different surface quality exhibited by the corresponding AFM images (Fig. 2, bottom panel). As seen from the GISAXS patterns (Fig.4), the NIL polymer gratings exhibit a concentration of the scattered intensity at lower $\omega$ values in comparison with that of the stamps which is more homogeneously distributed along the semicircle. This is also reflected in Fig.6. Modeling calculations have shown that this type of scattering can be simulated by considering one-dimensional crystals consisting on boxes of a certain length (Rueda et al., 2012). The length of the box controls the shape of the scattering pattern changing from the characteristic semicircle for large box lengths, to a rod-like pattern for shorter ones. Thus, in a first approach we propose to attribute this effect to a reduction of the effective length of the lines which diffract coherently, as the amount of defects on the line increases. As mentioned before, 
for the NIL polymer gratings, besides the characteristic scattering on semicircle, the patterns shown in Fig. 4 (bottom panel) exhibit significant scattering around the region of the Yoneda peak. It is known that at exit angles $\alpha$ equal to the critical one there is an amplification of the scattering which indicates the presence of diffuse scattering (Yoneda, 1963). Fig. 7 represents the scattered intensity along the vertical direction, $\alpha$ cut, at the position of the first scattering maximum of the GISAXS patterns shown in Fig. 4d, 4e, and 4f (continuous lines) for polymer NIL. For the sake of comparison the intensity of the cuts, corresponding to the stamps (Fig. 4a, 4b and 4c), has also been included (dotted lines). The exit angle $\alpha$ has been normalized to the experimental reflection angle of every sample. The excess of scattering around the Yoneda peak detected in the GISAXS patterns for the NIL samples can be visualized in Fig.7. In addition to the main intensity of the spot at the reflection angle, a remarkable diffuse scattering is observed for the NIL polymer gratings in contrast to the near flat background observed for the silicon stamps. It is also worth to notice the broadening of the main intensity peak for NIL polymer gratings in relation to that for stamps. This broadening can be attributed to a decrease of the length the lines diffracting coherently and it is in accordance with the presence of defects in the NIL polymer grating surface. Fig. 8 shows the ratio between the intensity at the maximum of the Yoneda angle region, $\alpha_{y}$, to that of the spot at the reflection angle, $\alpha_{i}$ which equals the incidence angle. There is a clear correlation of this magnitude with the amount of defects per line as estimated by image analysis (Fig.3).

\section{Conclusion.}

GISAXS can be used in an attempt to characterize structurally the replica quality of polymer gratings prepared by thermal Nanoimprint Lithography. GISAXS patterns of NIL polymer gratings with different line quality present characteristic features that, in a first approximation, can be associated with the level of defects per line. Both stamps and NIL polymer gratings exhibit the characteristic semicircle-like GISAXS patterns with a radius corresponding to the incidence angle used. However NIL polymer gratings with defective lines present GISAXS patterns with higher intensities around low $\omega$ angles and significant diffuse scattering in the region of the Yoneda peak as compared to the GISAXS patterns of the corresponding stamps. Taking into account our previous 
modeling calculations, which considers the gratings as one-dimensional crystals consisting of boxes of a certain length, we can attribute this effect to a reduction of the effective length of the lines which diffract coherently as the amount of defects on the line increases. Although the present study can be considered as an initial step to characterize NIL patterned polymers by GISAXS and it remains at a qualitative level, we think that the reported effects have potential to be implemented in the assessment of replica quality of the NIL process.

\section{Acknowledgements}

The financial support of MICINN through CTQ2010-15680 MAT2011-23455 and MAT2012-33517 is gratefully acknowledged. E.R. and I. M-F. thank MICINN, Spain, for a "Ramón y Cajal " contract (RYC-2011-08069) and for a FPI fellowship respectively. M.S. and D.-T thank CSIC for the tenure of JAE fellowships and the Fondo Social Europeo (FSE) for co-financing the JAE program. We are indebted to J. Perlich for outstanding beamline support.

\section{References}

Baumbach, T. \& Lubbert, D. (1999). Journal of Physics D-Applied Physics 32, 726-740.

Cecchini, M., Signori, F., Pingue, P., Bronco, S., Ciardelli, F. \& Beltram, F. (2008). Langmuir 24, 12581-12586.

Chandross, M. \& Grest, G. S. (2012). Langmuir 28, 1049-1055.

Chen, D., Zhao, W. \& Russell, T. P. (2012). ACS Nano 6, 1479-1485.

Dirckx, M. E. \& Hardt, D. E. (2011). Journal of Micromechanics and Microengineering 21, 085024.

Fernández Cuesta, I. (2009). Ph D Thesis, Universitat Autonoma de Barcelona, Barcelona.

Hammersley, A. (2004). http://www.esrf.eu/computing/scientific/FIT2D/.

Hernandez, J. J., Rueda, D. R., Garcia-Gutierrez, M. C., Nogales, A., Ezquerra, T. A., Soccio, M., Lotti, N. \& Munari, A. (2010). Langmuir 26, 10731-10737.

Hlaing, H., Lu, X. H., Nam, C. Y. \& Ocko, B. M. (2012). Small 8, 3443-3447.

Hu, Z. J. \& Jonas, A. M. (2010). Soft Matter 6, 21-28.

Hu, Z. J., Tian, M. W., Nysten, B. \& Jonas, A. M. (2009). Nature Materials 8, 62-67.

Lee, L. J. (2006). Annals of Biomedical Engineering 34, 75-88.

Lochbihler, H. (2009). Optics Express 17, 12189-12196.

Mayer, A. C., Scully, S. R., Hardin, B. E., Rowell, M. W. \& McGehee, M. D. (2007). Materials Today 10, 28-33.

Meier, R., Birkenstock, C., Palumbiny, C. M. \& Muller-Buschbaum, P. (2012a). Phys. Chem. Chem. Phys. 14, 15088-15098.

Meier, R., Chiang, H. Y., Ruderer, M. A., Guo, S. A., Korstgens, V., Perlich, J. \& MüllerBuschbaum, P. (2012b). Journal of Polymer Science Part B-Polymer Physics 50, 631641.

Metzger, T. H., Haj-Yahya, K., Peisl, J., Wendel, M., Lorenz, H., Kotthaus, J. P. \& Cargill, G. S. (1997). Journal of Applied Physics 81, 1212-1216. 
Mikulik, P., Jergel, M., Baumbach, T., Majkova, E., Pincik, E., Luby, S., Ortega, L., Tucoulou, R., Hudek, P. \& Kostic, I. (2001). Journal of Physics D-Applied Physics 34, A188-A192.

Mills, C. A., Escarre, J., Engel, E., Martinez, E., Errachid, A., Bertomeu, J., Andreu, J., Planell, J. A. \& Samitier, J. (2005). Nanotechnology 16, 369-375.

Müller-Buschbaum, P. (2006). High-resolution grazing incidence small angle X-ray scattering: Investigation of micrometer sized structured polymer films, Vol. 132, PROGRESS IN COLLOID AND POLYMER SCIENCE : Characterization of Polymer Surfaces and Thin Fllms, edited by K. Grundke, M. Stamm \& H. J. Adler, pp. 23-32. BERLIN: SPRINGERVERLAG.

Müller-Buschbaum, P. (2009). Applications of Synchrotron Light to Scattering and Diffraction in Materials and Life Sciences

Vol. 776, Lecture Notes in Physics

edited by T. A. Ezquerra, M. C. García-Gutiérrez, A. Nogales \& M. Gómez, Berlin: SpringerVerlag.

Perlich, J., Rubeck, J., Botta, S., Gehrke, R., Roth, S. V., Ruderer, M. A., Prams, S. M., Rawolle, M., Zhong, Q., Korstgens, V. \& Muller-Buschbaum, P. (2010). Rev. Sci. Instrum. 81, 105105.

Roth, S. V., Dohrmann, R., Dommach, M., Kuhlmann, M., Kroger, I., Gehrke, R., Walter, H., Schroer, C., Lengeler, B. \& Muller-Buschbaum, P. (2006). Rev. Sci. Instrum. 77, 085106.

Rowland, H. D., Sun, A. C., Schunk, P. R. \& King, W. P. (2005). Journal of Micromechanics and Microengineering 15, 2414-2425.

Rueda, D. R., Martin-Fabiani, I.Soccio, M. A., N.Perez-Murano, F.Rebollar, E.Garcia-Gutierrez, C.Castillejo, M. \& Ezquerra, T. A. (2012). Journal of Applied Crystallography 45, 10381045.

Schift, H. (2008). Journal of Vacuum Science \& Technology B 26, 458-480.

Timmann, A., Dohrmann, R., Schubert, T., Schulte-Schrepping, H., Hahn, U., Kuhlmann, M., Gehrke, R., Roth, S. V., Schropp, A., Schroer, C. \& Lengeler, B. (2009). Rev. Sci. Instrum. 80, 046103.

Wernecke, J., Scholze, F. \& Krumrey, M. (2012). Rev. Sci. Instrum. 83, 103906.

Yan, M. \& Gibaud, A. (2007). J. Appl. Crystallogr. 40, 1050-1055.

Yoneda, Y. (1963). Physical Review 131, 2010-2013. 


\section{Figure captions}

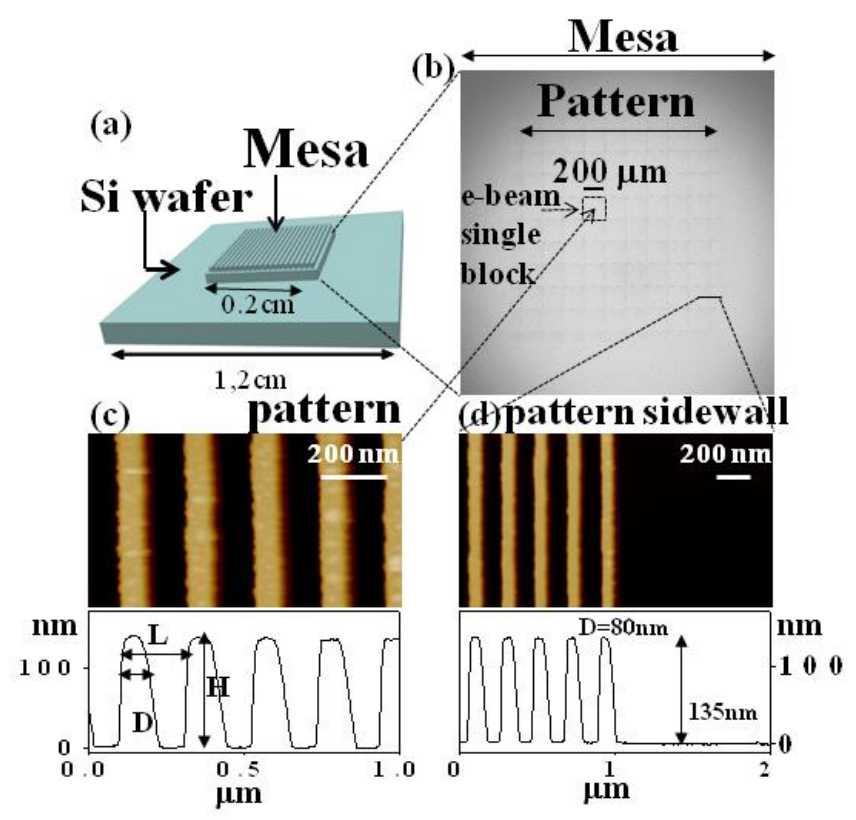

Fig.1. (a) Scheme of a mesa stamp. (b) SEM image of a typical mesa area patterned by e-beam lithography where the single e-beam blocks can be visualized. (c) AFM height image of the stamp grating. (d) AFM height image of the sidewall of the patterned area protruding from the mesa. Corresponding height profiles are included at the bottom. 


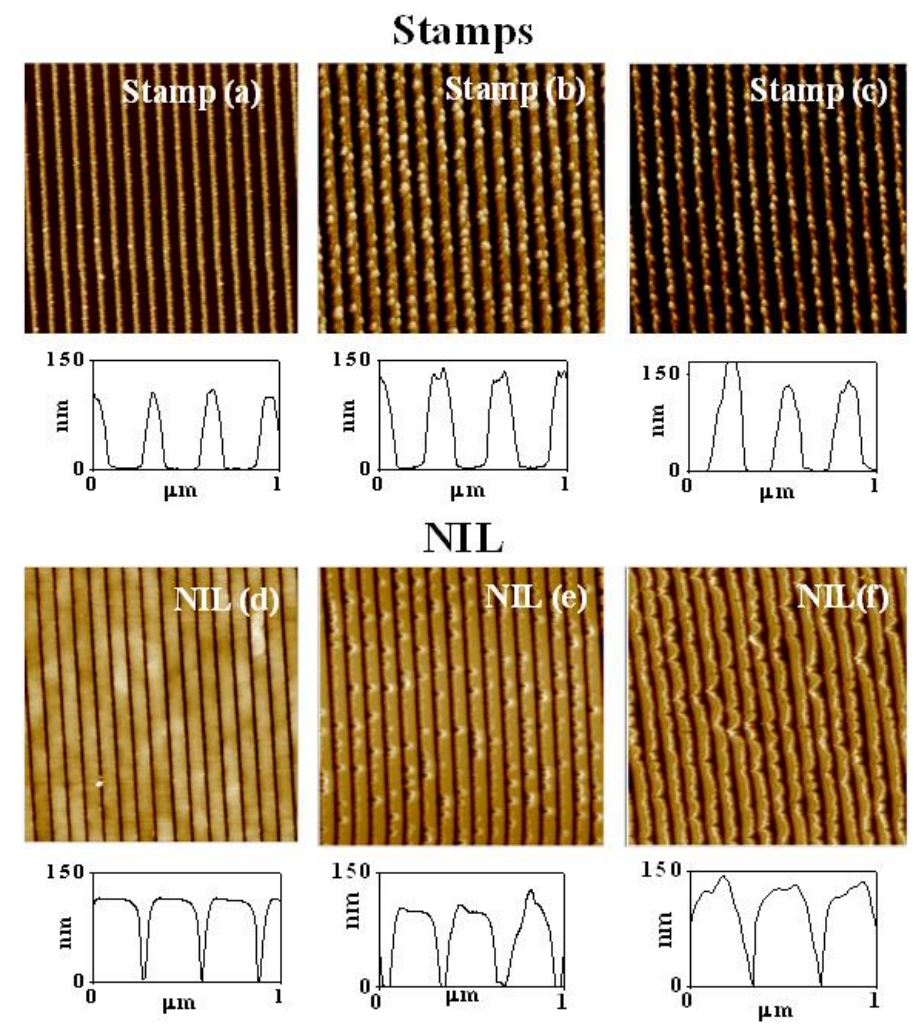

Fig.2. AFM height images $\left(5 \times 5 \mu \mathrm{m}^{2}\right)$ of: (Upper panel) different silicon stamps of similar pitch $(300 \mathrm{~nm})$ and different line width $\left(\mathrm{D}_{\text {stamp }}\right)$ and line height $\left(\mathrm{H}_{\text {stamp }}\right)$. (Bottom panel) Corresponding PBAC gratings prepared with the stamps. Height profile of both stamps and polymer gratings along a cut perpendicular to the lines is shown below.

(d)

(e)

(f)
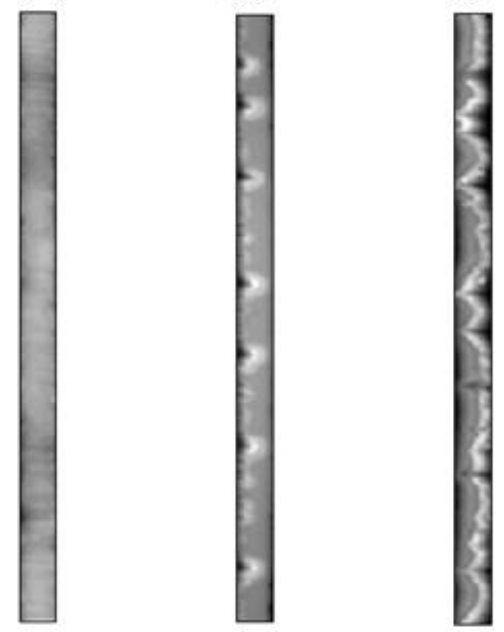

$0.5 \pm 0.1 \% \quad 5 \pm 1 \% \quad 8 \pm 1 \%$ 
Fig.3. AFM height images ( $5 \mu \mathrm{m}$ length) of single lines corresponding to the PBAC NIL gratings shown in Fig.2d, 2e and 2f. Bottom labels indicate the estimates of the defect level as calculated from the ratio between dark (defective) and total line area.

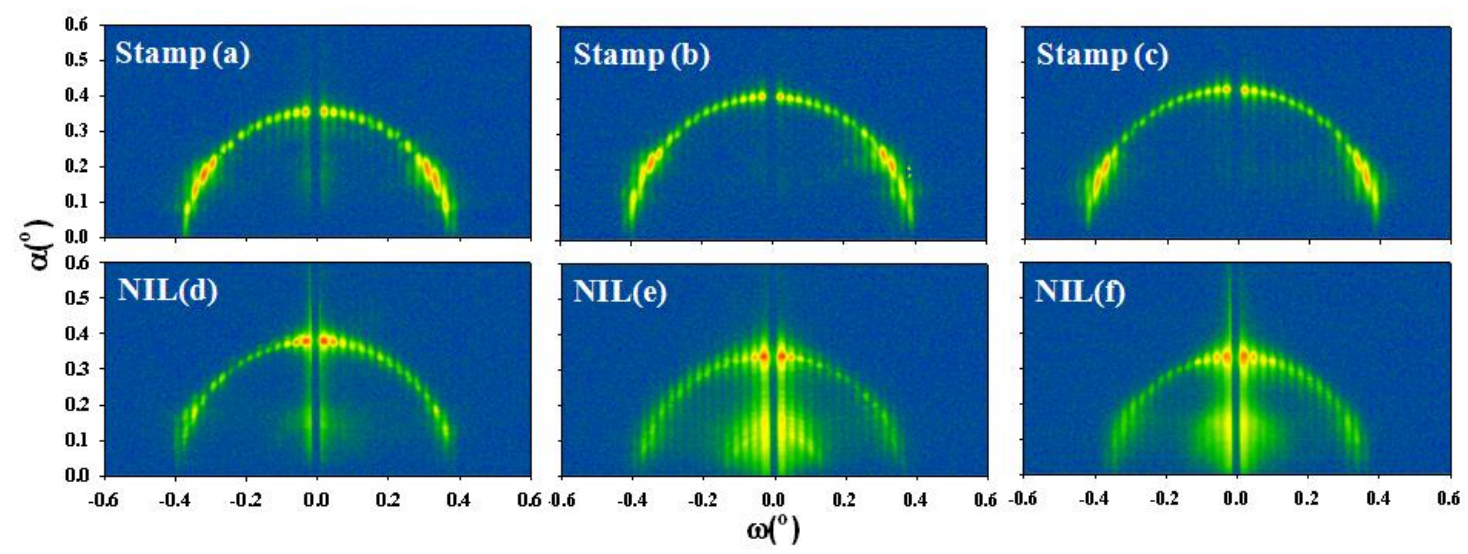

Fig.4. GISAXS patterns corresponding to the gratings shown in Fig. 2. Top panel: Silicon stamps. Bottom panel: NIL polymer (PBAC) gratings.

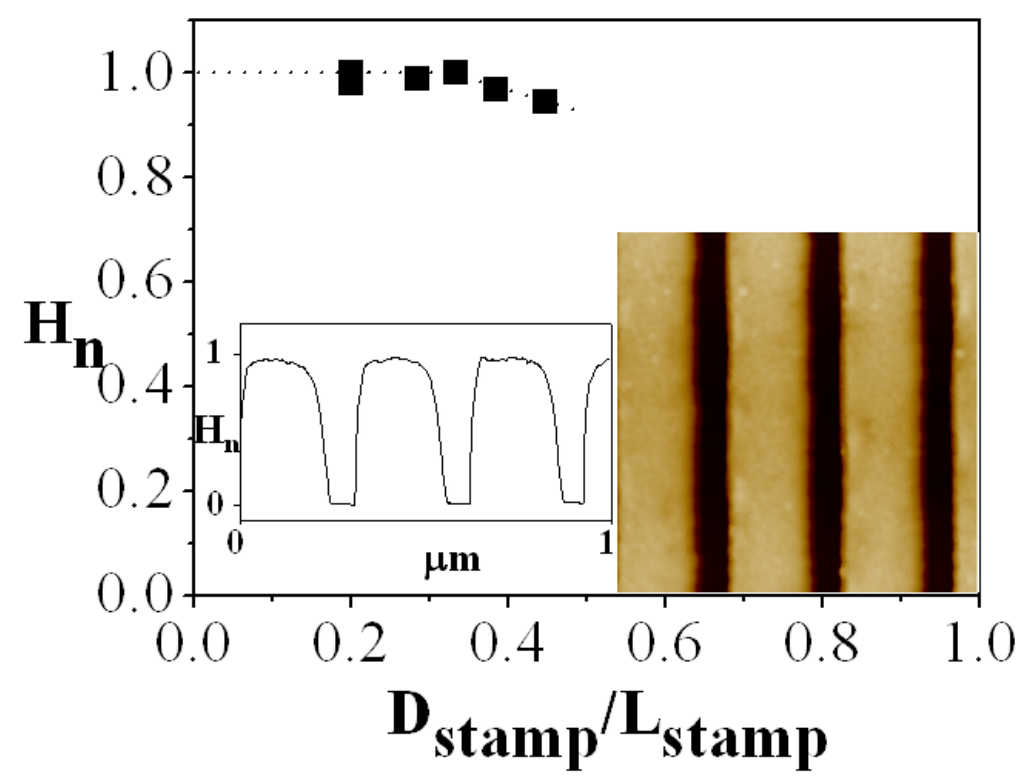

Fig.5. Ratio $\left(\mathrm{H}_{\mathrm{n}}=\mathrm{H}_{\mathrm{nil}} / \mathrm{H}_{\text {stamp }}\right)$ between the heights of the NIL polymer gratings $\left(\mathrm{H}_{\text {nil }}\right)$ to those of the corresponding stamps $\left(\mathrm{H}_{\text {stamp }}\right)$ as a function of the ratio of the indenter width to the pitch in the stamps $\left(D_{\text {stamp }} / L_{\text {stamp }}\right)$ for $L_{\text {stamp }}=300 \mathrm{~nm}(\mathbf{\square})$. The dashed line is a guide for the eyes. As an example, the inset shows an AFM height image $\left(1 \mathrm{x} 1 \mu \mathrm{m}^{2}\right)$ 
and the corresponding height profile normalized with respect to the height of the stamp, of a NIL polymer grating with a filling factor of 0.39 .

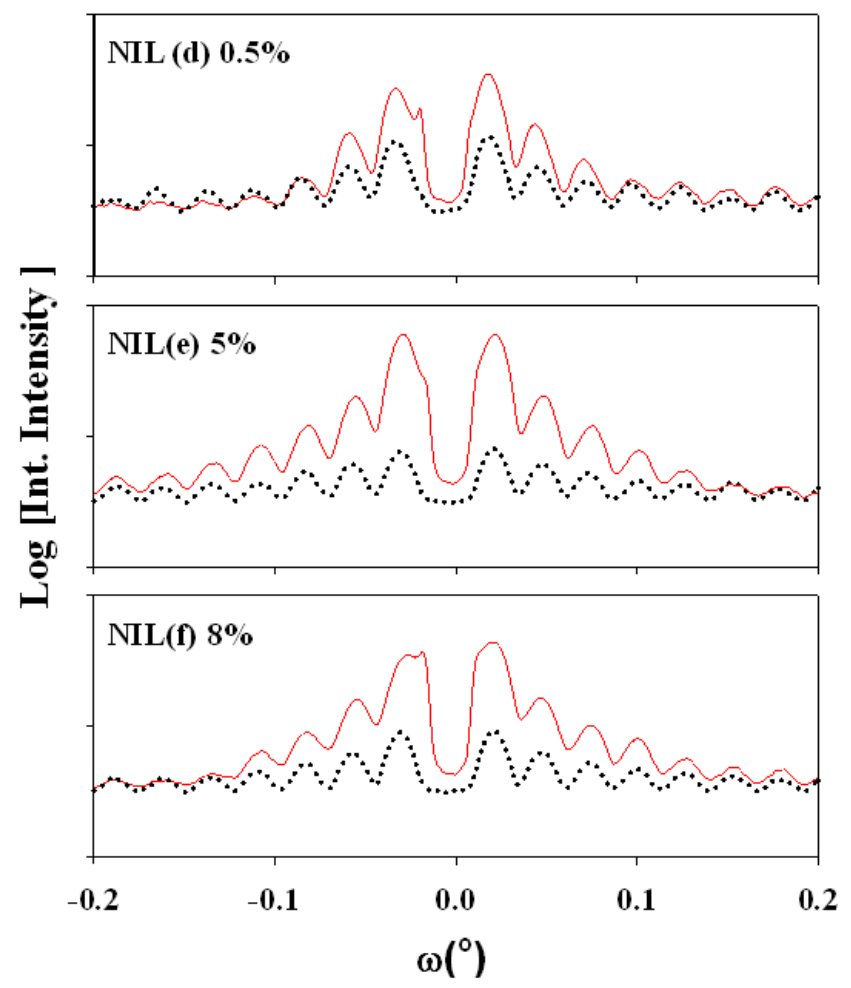

Fig. 6. Total integrated intensity along the vertical direction of the GISAXS patterns corresponding to the NIL polymer gratings (red continuous lines) shown in Fig.4d, 4e and $4 \mathrm{f}$ respectively. The dotted lines represent the total integrated intensity of the corresponding stamps (Fig. 4a, $4 \mathrm{~b}$ and $4 \mathrm{c}$ ). The labels indicate the amount of defects as estimated by image analysis (Fig.3). 


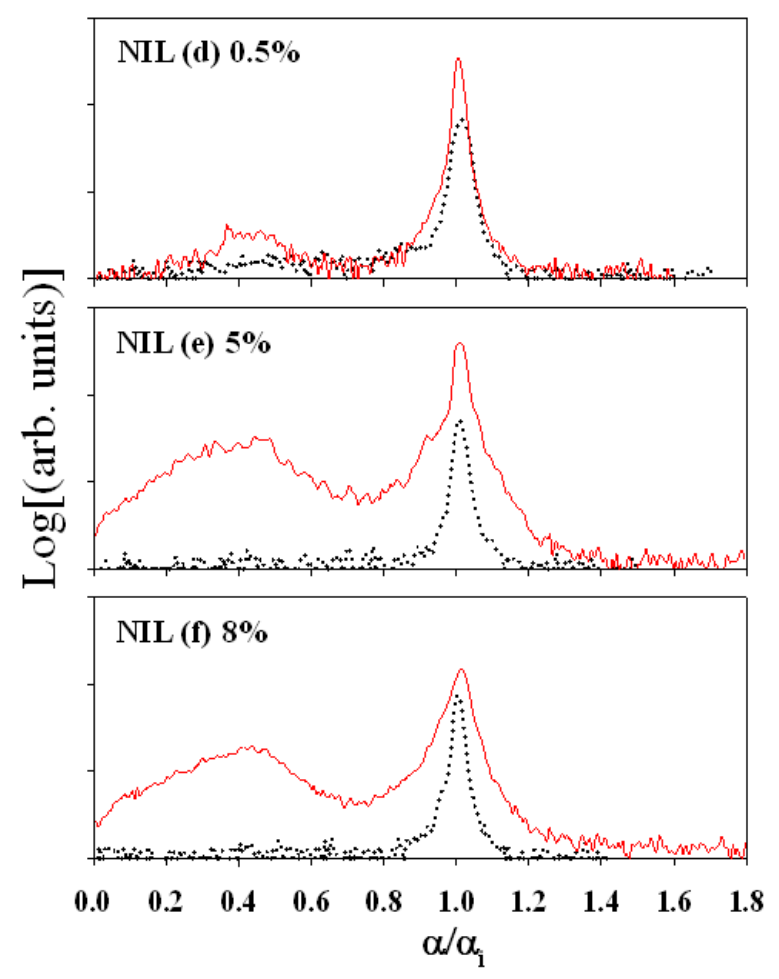

Fig. 7. Intensity along the vertical direction of the GISAXS patterns corresponding to the first scattering maximum of the NIL polymer gratings (red continuous lines) shown in Fig.4d, 4e and 4f respectively. Dotted lines represent the intensity of the $\alpha$-cuts corresponding to the stamps (Fig. 4a, 4b and 4c). The exit angle $\alpha$ has been normalized to the angle of incidence, $\alpha_{i}$. Labels indicate the amount of defects as estimated by image analysis (Fig.3). 


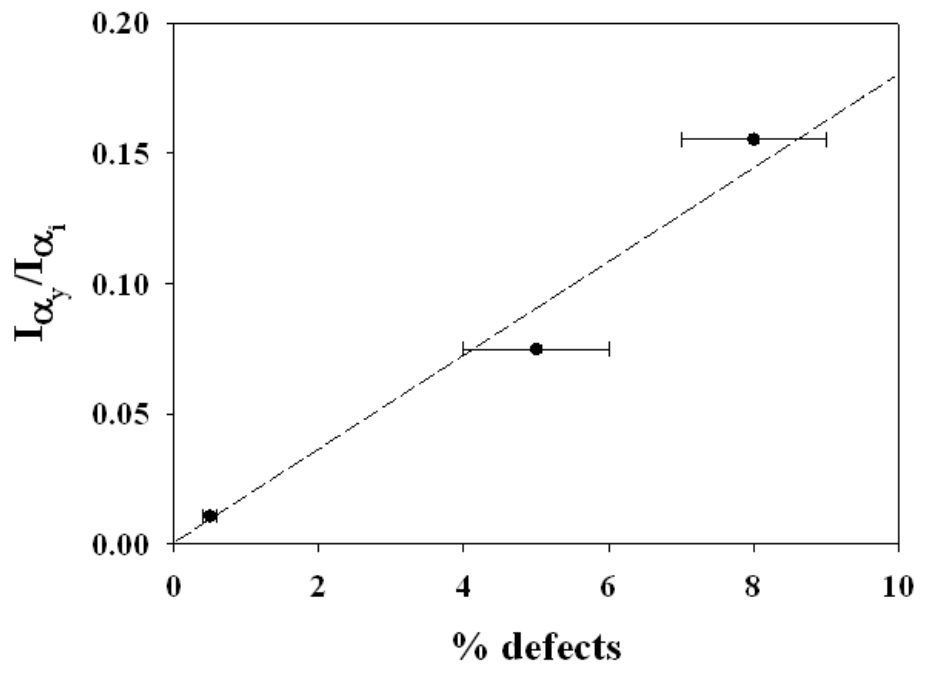

Fig. 8. Intensity ratio between the maximum in the Yoneda angular region, $\alpha_{y}$, and that of the reflection angle, $\alpha_{i}$, as a function of the amount of defects per line as estimated by image analysis (Fig.3). 